\title{
Zum Mechanismus der Hydroxylierungen mit molekularem Sauerstoff und Zinn(II)-Komplexen *
}

\author{
Volker Ullrich und Hansjürgen Staudinger
}

Physiologisch-chemisches Institut der Justus Liebig-Universität Gießen

(Z. Naturforschg. 24 b, 583-588 [1969] ; eingegangen am 25. Oktober 1968)

\begin{abstract}
Reduction of molecular oxygen by a stannous phosphate complex in aqueous solution leads to a new species of active oxygen capable of hydroxylating aliphatic and aromatic compounds. The hydroxylation reactions are shown to require two electrons per oxygen molecule reduced. The oxygen atom of the hydroxyl group is completely derived from molecular oxygen. This new mechanism has been proposed as a model for mixed function oxygenases.
\end{abstract}

Mischfunktionelle Oxygenierungen verlaufen nach der zuerst von MASON ${ }^{1}$ aufgestellten Gleichung:

$$
\mathrm{RH}+\mathrm{DH}_{2}+\mathrm{O}_{2} \rightarrow \mathrm{ROH}+\mathrm{D}+\mathrm{H}_{2} \mathrm{O} .
$$

$\mathrm{RH}=$ organisches Substrat, $\mathrm{DH}_{2}=$ Wasserstoffdonator.

Über den entscheidenden Schritt dieser komplexen Reaktion, die Aktivierung des Sauerstoffs, ist bisher nur wenig bekannt.

Auch geeignete Modellsysteme fehlen, da in den bisher beschriebenen Hydroxylierungs-Systemen mit molekularem Sauerstoff und reduzierten Schwermetallionen mindestens zwei chemisch verschiedene Mechanismen nebeneinander ablaufen ${ }^{2-4}$. Zum überwiegenden Teil erfolgt die Hydroxylierung in diesen Systemen durch OH-Radikale, die bei der Einelektronen-Reduktion von intermediär gebildetem Wasserstoffperoxid entstehen. Daneben konnten wir noch einen zweiten Hydroxylierungs-Mechanismus nachweisen, der sich von den Hydroxylierungen durch OH-Radikale in charakteristischer Weise unterscheidet. Die Hydroxylierung von Aromaten und Substanzen mit Doppelbindungen erfolgt nach diesem zweiten Mechanismus nicht wie durch $\mathrm{OH}$-Radikale elektrophil, sondern statistisch gleich an allen $\mathrm{CH}$ Bindungen ${ }^{3,5}$. Außerdem werden, ebenfalls im Gegensatz zu den Reaktionen der OH-Radikale, aliphatische Verbindungen glatt hydroxyliert ${ }^{5}$. Wegen sei-

* Auszugsweise vorgetragen auf dem Internationalen Symposium über "Microsomes and Drug Oxidations" in Bethesda, Md., USA, 1968.

1 H. S. Mason, Advances in Enzymol. 19, 79 [1957].

2 HJ. Staudinger u. V. Ullrich, Z. Naturforschg. 19 b, 409 [1964].

3 Hj. Staudinger u. V. Ullrich, Z. Naturforschg. 19 b, 877 [1964].

4 V. Ullrich, D. Hey, HJ. Staudinger, H. Büch u. W. Rummel, Biochem. Pharmacol. 16, 2237 [1967]. ner großen Ähnlichkeit mit den Carben-Reaktionen wurde dieser neue Mechanismus von uns als „Oxen“oder "Oxenoid-Hydroxylierung" bezeichnet ${ }^{6,7}$.

OH-Radikale können als der "aktive Sauerstoff" bei enzymatischen Hydroxylierungen aus verschiedenen Gründen ausgeschlossen werden ${ }^{7,8}$. Dagegen zeigt der Oxenoid-Mechanismus weitgehende Analogien zu dem Hydroxylierungs-Mechanismus der Monooxygenasen ${ }^{4,7}$.

Es blieb noch zu beweisen, daß bei dem Modellmechanismus genau wie bei den enzymatischen Reaktionen zwei Elektronen zur Aktivierung des Sauerstoffs verwendet werden, und daß das eingeführte Sauerstoffatom aus dem molekularen Sauerstoff stammt.

Diese und einige weitere Versuche zur Aufklärung des Oxen-Mechanismus wurden mit dem System Zinn (II)/Phosphat/Sauerstoff durchgeführt, in dem nach unseren früheren Befunden ausschließlich der Oxen-Mechanismus auftritt ${ }^{6}$.

\section{Material und Methoden}

Dodecadeuterocyclohexan $\left(\mathrm{Uvasol}^{\circledR}\right.$, min. 99-proz.), Cyclohexan $\left(\mathrm{Uvasol}^{\circledast}\right.$ ), und F o l in s Reagenz zur Phenolbestimmung wurden von der Firma E. Merck AG, Darmstadt, bezogen. Das bei den Isotopenexperimenten eingesetzte Wasser mit $5 \% \mathrm{H}_{2}{ }^{18} \mathrm{O}$ stammte von der Firma Buchler \& Co, Braunschweig. Alle übrigen Reagenzien wurden p.a. verwendet.

5 V. Ullrich, J. Wolf, E. Amadori u. HJ. Staudinger, Hoppe-Seyler's Z. physiol. Chem. 349, 85 [1968].

6 V. Ullrich, E. Amadori u. HJ. Staudinger, Z. Naturforschg. 22 b, 226 [1967].

7 V. Ullrich u. HJ. Staudinger, in: Biological and chemical aspects of oxygenases, K. BLOCH and O. HAYAISHI, Eds., Maruzen Company, Tokio 1966.

8 V. Ullrich and HJ. Staudinger, on: International Symposium on Microsomes and Drug Oxidations, Bethesda, Md., 1968, im Druck. 


$$
\text { Versuche mit }{ }^{18} \mathrm{O}_{2}
$$

${ }^{18} \mathrm{O}_{2}$-angereicherter Sauerstoff wurde durch Elektrolyse von 5-proz. $\mathrm{H}_{2}{ }^{18} \mathrm{O}$ hergestellt. Dazu diente eine modifizierte Apparatur nach SAMUeL ${ }^{9}$. Vor der Elektrolyse wurde das Wasser mit metallischem Natrium auf eine $\mathrm{NaOH}-\mathrm{Konzentration}$ von $2-n$. gebracht und anschließend mit $18 \mathrm{~V}$ bei $0,6 \mathrm{~A}$ elektrolysiert. Der entwickeite Sauerstoff wurde in einem Vorratsgefäß乃 unter Quecksilber aufgefangen. Danach wurde die gesamte Apparatur mit dem $50 \mathrm{ml}$ fassenden Reaktionsgefäß mit superreinem Stickstoff gespült und unter Durchleiten von Stickstoff die Acetanilid-Lösung mit der anaeroben Lösung des Zinn (II) -phosphat-Komplexes (zusammen $10,0 \mathrm{ml}$ mit den in Tab. 1 angegebenen Konzentrationen) durch den seitlichen Ansatz eingefüllt. Nach zweimaligem Evakuieren und Spülen mit Stickstoff wurde die evakuierte Apparatur mit dem Sauerstoff unter geringem Überdruck gefüllt und damit die Reaktion unter Rühren gestartet. Während der Reaktion wurden aus dem Reaktionsraum zwei Gasproben über ein $\mathrm{P}_{2} \mathrm{O}_{5}$-Trockenrohr entnommen. Nach zwei Stdn. war die Reaktion vollständig abgelaufen. Die Trennung der $N$-Acetylaminophenole aus fünf Ansätzen erfolgte durch zweimalige Dünnschichtchromatographie an Kieselgel in einem Laufmittel aus Benzol (90), Methanol (16) und Eisessig (8) (v/v/v). Die mit Methylenchlorid extrahierten Kieselgelzonen wurden von dem Applikationslabor der Firma Bell \& Howell, Friedberg (Hessen), massenspektrometrisch analysiert.

\section{Bestimmung der Stöchiometrie}

Die Messung der Sauerstoffaufnahme erfolgte manometrisch in der für die ${ }^{18} \mathrm{O}$-Experimente verwendeten Apparatur, die durch ein graduiertes Vorratsgefäß ergänzt wurde. In den Reaktionskolben wurden $10 \mathrm{ml}$ n-Hexanol gefüllt und durch den zeitlichen Ansatz eine genau abgewogene Menge Zinn(II)-chlorid-Dihydrat gegeben und sofort verschlossen. Im Niveaugefäß ließ sich der Verbrauch des Sauerstoffs verfolgen. Am Ende der Reaktion wurde die Differenz bestimmt und das Normalvolumen des $\mathrm{O}_{2}$ auf die iodometrisch bestimmten Reduktionsäquivalente des eingesetzten Zinnchlorids bezogen. Als Kontrolle diente ein Ansatz ohne Hexanol mit $10 \mathrm{ml}$ Wasser in dem die theoretisch zu fordernde Stöchiometrie von 4. Elektronen pro $\mathrm{O}_{2}$-Molekül gefunden wurde.

\section{Belichtungsversuche}

$50 \mathrm{ml}$ eines Ansatzes mit den in Tab. 1 angegebenen Konzentrationen wurden in einem 250-ml-Becherglas mit dem Magnetstab gerührt. Je ein Ansatz wurde dabei im Abstand von $30 \mathrm{~cm}$ mit einer $300 \mathrm{~W}$ Lampe (Osram „Concentra“) bestrahlt, während die Kontrolle völlig verdunkelt wurde. In Abständen von fünf Min. wurden beiden Ansätzen je 2,0 ml entnommen, mit $0,1 \mathrm{ml} 5-n . \mathrm{NaOH}$ gestoppt und die Gesamtmenge an gebildeten Phenolen mit dem Folin-Reagenz nach der Methode von $\mathrm{KRISCH}$ et al. ${ }^{10}$ bestimmt.

\section{Bestimmung der N-Acetylaminophenole}

Die Aufarbeitung der Ansätze sowie die dünnschichtchromatographische Bestimmung der $N$-Acetylaminophenole erfolgte wie früher von uns angegeben ${ }^{5}$.

\section{Ergebnisse}

In allen bisher beschriebenen Systemen zur Hydroxylierung mit molekularem $\mathrm{O}_{2}$ und reduzierten Schwermetallkomplexen sind sowohl OH-Radikale als auch der Oxen-Mechanismus an der Hydroxylierung beteiligt. Besonders gut läßt sich das an Hand des $o: m: p:-$ Verhältnisses bei der Hydroxylierung aromatischer Verbindungen mit Substituenten erster Ordnung zeigen.

\begin{tabular}{|c|c|c|c|c|c|c|c|c|c|c|c|c|}
\hline \multirow{3}{*}{$\begin{array}{c}t \\
{\left[\begin{array}{c}\left.{ }^{\circ} \mathrm{C}\right] \\
0\end{array}\right.} \\
0\end{array}$} & \multicolumn{3}{|c|}{$\begin{array}{c}\text { System 1 } \\
\mathrm{Fe}^{2 \oplus / \text { EDTA } / O_{2}}\end{array}$} & \multirow{3}{*}{$\begin{array}{c}\text { Ausbeute } \\
{[\mu \mathrm{Mol}]} \\
0,68\end{array}$} & \multicolumn{3}{|c|}{ 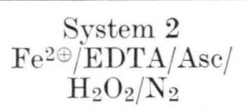 } & \multirow{2}{*}{$\begin{array}{c}\text { Ausbeute } \\
{[\mu \mathrm{Mol}]}\end{array}$} & \multicolumn{3}{|c|}{$\begin{array}{c}\text { System } 3 \\
\mathrm{Sn}^{2+} / \mathrm{HPO}_{4}^{2-} / \mathrm{O}_{2}\end{array}$} & \multirow{2}{*}{$\begin{array}{c}\text { Ausbeute } \\
{[\mu \mathrm{Mol}]}\end{array}$} \\
\hline & $\begin{array}{c}0 \\
{[\%]}\end{array}$ & & & & $\begin{array}{c}o \\
{[\%]}\end{array}$ & & $p$ & & {$\left[\begin{array}{c}o \\
{[\%}\end{array}\right]$} & & $p$ & \\
\hline & 28 & 24 & 48 & & 48 & 3 & 49 & 0,89 & 36 & 35 & 29 & 0,41 \\
\hline 25 & 36 & 14 & 50 & 0,64 & 47 & 3 & 50 & 0,84 & 34 & 44 & 22 & 0,36 \\
\hline 50 & 40 & 9 & 51 & 0,78 & 43 & 3 & 54 & 0,78 & 34 & 41 & 25 & 0,62 \\
\hline 100 & 45 & 5 & 50 & 0,79 & 44 & 3 & 53 & 0,77 & 35 & 35 & 30 & 0,72 \\
\hline
\end{tabular}

Tab. 1. Temperaturabhängigkeit der $o: m: p$-Relationen bei der Hydroxylierung von Acetanilid in Modellsystemen. System 1: $20 \mu \mathrm{Mol} \mathrm{FeSO}{ }_{4} \cdot 7 \mathrm{H}_{2} \mathrm{O}, 30 \mu \mathrm{Mol}$ EDTA, $40 \mu \mathrm{Mol}$ Acetanilid in 2,0 ml 0,1-m. Acetatpuffer pH 5,8. $40 \mathrm{Min}$. unter Schütteln inkubieren. System 2: $5 \mu$ Mol FeSO $4 \cdot 7 \mathrm{H}_{2} \mathrm{O}, 7,5 \mu \mathrm{Mol}$ EDTA, $30 \mu \mathrm{Mol}$ Ascorbinsäure, $40 \mu \mathrm{Mol}$ Acetanilid, $3 \mu \mathrm{Mol}$ $\mathrm{H}_{2} \mathrm{O}_{2}$, in $2,0 \mathrm{ml} \mathrm{0,1-m}$. Acetatpuffer $\mathrm{pH}$ 5,8. Inkubationszeit nach $\mathrm{H}_{2} \mathrm{O}_{2}$-Zugabe: 5 Min. unter Durchperlen von Stickstoff.

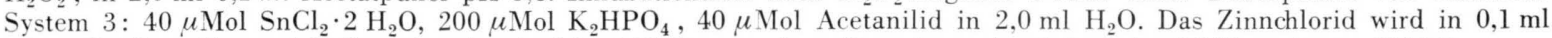
$0,1-n . \mathrm{H}_{2} \mathrm{SO}_{4}$ gelöst und Kaliumphosphat in $0,3 \mathrm{ml} \mathrm{H}_{2} \mathrm{O}$ zugegeben. Danach werden $1,6 \mathrm{ml}$ der $0,025-m$. Acetanilid-Lösung zugefügt. Das pH wird auf 10,5 eingestellt. Es wird 40 Min. unter Schütteln an der Luft inkubiert.

9 D. Samuel, in: Oxygenases, Academic Press, New YorkLondon 1962, S. 31 .
10 K. Krisch u. HJ. Staudinger, Biochem. Z. 334, 312 [1961]. 
OH-Radikale reagieren streng elektrophil ${ }^{11,12}$ und bevorzugen daher die ortho- und para-Stellung, während der Oxen-Mechanismus zu einer mehr statistischen Verteilung der Phenole führt. In einem System, in dem beide Mechanismen vorkommen, ist der relative Anteil beider Mechanismen von der Temperatur abhängig ${ }^{3,4}$. Mit steigender Temperatur wird der Oxen-Mechanismus zugunsten der Hydroxylierung durch $\mathrm{OH}-$ Radikale zurückgedrängt.

Man beobachtet also eine Zunahme der Selektivität des Angriffs, während bei einem einheitlichen Mechanismus allenfalls eine Abnahme der Selektivität bei Temperaturerhöhung erwartet werden kann ${ }^{13}$.

In Tab. 1 ist die Temperaturabhängigkeit der isomeren $N$-Acetylaminophenole für das System Zinn (II) $/$ Phosphat $/ \mathrm{O}_{2}$ im Vergleich zu den schon früher beschriebenen Systemen Eisen (II) $/$ EDTA $/ \mathrm{O}_{2}$ und Eisen(II)/EDTA/Ascorbinsäure $/ \mathrm{N}_{2} \quad(=\mathrm{OH}$ Radikale) aufgeführt.

Bei der Hydroxylierung von Acetanilid im Zinnsystem ist keine signifikante Verschiebung der $o: m: p$-Relationen bei Temperaturänderung festzustellen. Das läßt den Schluß zu, daß wie in dem modifizierten Fenton-System auch ein einheitlicher aber anderer Hydroxylierungs-Mechanismus auftritt.

Der Grund für die Sonderstellung des Zinnsystems läßt sich durch einen einfachen Versuch veranschaulichen (Tab. 2) :

Durch einen Zusatz von Wasserstoffperoxid entstehen in dem System $\mathrm{Fe}^{2 \odot} / \mathrm{EDTA}_{/} \mathrm{O}_{2}$ durch Einelektronen-Reduktion $\mathrm{OH}$-Radikale, während der Zinn(II)-Komplex als Zweielektronendonator Wasserstoffperoxid sofort zu Wasser reduziert.
Aus der Kinetik des Zinnsystems geht hervor, daß die Hydroxylierung in einer Reaktion erster Ordnung in bezug auf $\mathrm{O}_{2}$ und Substrat verläuft (Abb. 1).

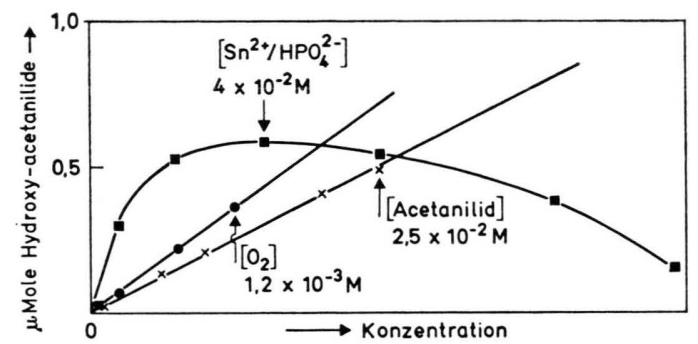

Abb. 1. Kinetik der Acetanilid-Hydroxylierung im System $\mathrm{Sn}^{2} \odot / \mathrm{HPO}_{4}{ }^{2 \odot} / \mathrm{O}_{2}$; Versuchsbedingungen: s. Tab. 1 .

Dagegen ist die Abhängigkeit der Hydroxylierung von dem Zinn(II) -phosphat-Komplex nicht linear; sie besitzt ein Optimum, da sie durch hohe Zinn(II) Konzentrationen gehemmt wird. Auch Zusätze anderer oxydierbarer Substanzen bewirken eine Hemmung der Hydroxylierung, da sie mit dem Substrat um den ,aktiven $\mathrm{O}_{2}$ “ konkurrieren (Tab. 3).

\begin{tabular}{lcc}
\hline \multicolumn{1}{c}{ Hemmstoff } & Konzentration & $\begin{array}{c}\text { Hemmung } \\
{[\%]}\end{array}$ \\
\hline Natriumsulfit & $2 \cdot 10^{-2} \mathrm{~m}$. & 74 \\
Kaliumbromid & $2 \cdot 10^{-2} \mathrm{~m}$. & 64 \\
Cyclohexandicarbonsäure & $2 \cdot 10^{-2} \mathrm{~m}$. & 55 \\
Dimethylsulfoxid & $2 \cdot 10^{-2} \mathrm{~m}$. & 62 \\
Ammoniumsulfat & $2 \cdot 10^{-2} \mathrm{~m}$. & 42 \\
Natriumnitrit & $2 \cdot 10^{-2} \mathrm{~m}$. & 88 \\
Mangan(II)-sulfat & $2 \cdot 10^{-3} \mathrm{~m}$. & 92 \\
\hline
\end{tabular}

Tab. 3. Hemmung der Acetanilid-Hydroxylierung im System $\mathrm{Sn}^{2 \odot} / \mathrm{HPO}_{4}{ }^{2 \odot} / \mathrm{O}_{2}$.

Versuchsbedingungen wie in Tab. 1 angegeben. Die Hemmung wurde kinetisch durch Messung der Gesamtphenole nach $\mathrm{KRISCH}$ et al. ${ }^{10}$ verfolgt.

\begin{tabular}{|c|c|c|c|c|c|c|c|c|}
\hline & \multicolumn{3}{|c|}{$\begin{array}{l}\text { System: Fe } \\
\text { Hydroxyacetanilide }\end{array}$} & \multirow{2}{*}{$\begin{array}{c}\oplus \oplus / \mathrm{P}_{2} \mathrm{O}_{7} 4 \ominus / \mathrm{O}_{2} \\
\text { rel. Ausbeuten } \\
{[\%]}\end{array}$} & \multicolumn{4}{|c|}{ System: $\mathrm{Sn}^{2 \oplus} / \mathrm{HPO}_{4}{ }^{2} \odot / \mathrm{O}_{2}$} \\
\hline & $\left.\begin{array}{c}o \\
{[\%}\end{array}\right]$ & $\begin{array}{c}m \\
{[\%]}\end{array}$ & $\begin{array}{c}p \\
{[\%]}\end{array}$ & & $\begin{array}{c}o \\
{[\%]}\end{array}$ & $\begin{array}{c}m \\
{[\%]}\end{array}$ & $\begin{array}{c}p \\
{[\%]}\end{array}$ & {$[\%]$} \\
\hline Kontrolle & 41 & 17 & 42 & 100 & 34 & 44 & 22 & 100 \\
\hline$+\mathrm{H}_{2} \mathrm{O}_{2}$ & 41 & 6 & 53 & 115 & - & - & - & 0 \\
\hline
\end{tabular}

Tab. 2. Einfluß von Wasserstoffperoxid auf die Hydroxylierung von Acetanilid.

$20 \mu \mathrm{Mol} \mathrm{FeSO} \cdot \cdot 7 \mathrm{H}_{2} \mathrm{O}, 60 \mu \mathrm{Mol} \mathrm{Na} \mathrm{P}_{2} \mathrm{O}_{7} \cdot 10 \mathrm{H}_{2} \mathrm{O}, 20 \mu \mathrm{Mol} \mathrm{H} \mathrm{O}_{2}$ in $2,0 \mathrm{ml} \mathrm{H} \mathrm{H}_{2} \mathrm{O}$, pH 10,5. Inkubationszeit 40 Min. unter Schütteln an der Luft. System $\mathrm{Sn}^{2 \oplus} / \mathrm{HPO}_{4}{ }^{2 \ominus} / \mathrm{O}_{2}$ wie in Tab. 1 angegeben.

11 R. O. C. Norman and G. K. Radda, Proc. chem. Soc. [London] 1962, 138.

12 L. M. Dorfman, A. Taub, and R. E. Büchler, J. chem. Physics 36, 3051 [1962].
13 J. Hine, Reaktivität und Mechanismus, S. 353, Thieme, Stuttgart 1960. 
Als besonders wirksame Hemmstoffe erweisen sich Nitrit- und Mangan(II) -ionen.

Es ist bekannt, daß die Autoxydation von Zinn(II) -Salzen durch Belichtung beschleunigt wird ${ }^{14}$. Dieser Effekt läßt sich bei dem Zinn(II) -phosphatKomplex nachweisen und ist bei der Diskussion zum Mechanismus der $\mathrm{O}_{2}$-Reduktion von Redeutung (Abb. 2).

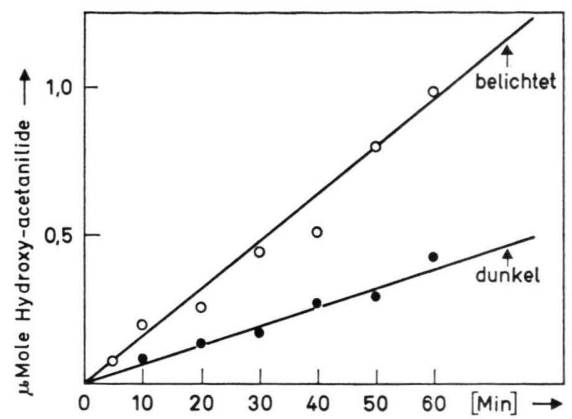

Abb. 2. Einfluß von Licht auf die Hydroxylierung von Acetanilid im System $\mathrm{Sn}^{2 \oplus} / \mathrm{HPO}_{4}{ }^{2} \odot / \mathrm{O}_{2}$; Konzentrationen wie in Tab. 1 angegeben. Versuchsbedingungen s. unter "Methoden“.

Ein weiterer Hinweis auf den Mechanismus des Systems war durch Messung des Isotopeneffektes bei der Hydroxylierung zu erwarten. Wir verwendeten dazu Cyclohexan und Brombenzol mit ihren entsprechenden vollständig deuterierten Derivaten als Substrate.

\begin{tabular}{lc}
\hline \multicolumn{1}{c}{ Substrat } & $k_{\mathrm{H}} / k_{\mathrm{D}}$ \\
\hline $\begin{array}{l}\text { Cyclohexan } \\
\text { Dodekadeutero-cyclohexan } \\
\text { Brombenzol }\end{array}$ & $1,28 \pm 0,04$ \\
Hexadeutero-brombenzol & $1,01 \pm 0,02$ \\
\hline
\end{tabular}

Tab. 4. Isotopeneffekte bei Hydroxylierungen im System $\mathrm{Sn}^{2 \oplus} / \mathrm{HPO}_{4}{ }^{2} \odot / \mathrm{O}_{2}$.

Das Ansatzvolumen beträgt $20 \mathrm{ml}$ mit den in Tab. 1 angegebenen Konzentrationen. $0,2 \mathrm{ml}$ des Substrates wurden als organische Phase zugegeben und die Mischung in verschlossenen Schliffgläsern horizontal geschüttelt. Cyclohexanol und Cyclohexanon wurden nach der Extraktion mit $5 \mathrm{ml}$ Essigester auf $0,2 \mathrm{ml}$ eingeengt und gaschromatographisch auf einer $2 \mathrm{~m}$ Polyäthylenglykol-1500-Säule bei $120^{\circ} \mathrm{C}$ getrennt. FIDDetektor, Gaschromatograph F 6, Perkin-Elmer. Die Bromphenole wurden aus dem sauren Ansatz mit $10 \mathrm{ml}$ Äther extrahiert und in $1 \mathrm{ml} 0,1-\mathrm{m}$. NaOH zurückgeschüttelt. Nach $\mathrm{Zu}$ gabe von $1,0 \mathrm{ml} \mathrm{Folins} \mathrm{Reagenz} \mathrm{(1:4} \mathrm{verd.)} \mathrm{und} 1,0 \mathrm{ml}$ $1-m . \mathrm{Na}_{2} \mathrm{CO}_{3}$ wurde die blaue Farbe nach 30 Min. bei $578 \mathrm{~nm}$ im Photometer gegen den Leerwert bestimmt.

${ }^{14}$ F. A. Cotton u. G. Wilkinson, Anorganische Chemie, Verlag Chemie, Weinheim/Bergstr. 1967, S. 449.
Für Brombenzol läßt sich kein Isotopeneffekt nachweisen. Da eine Substitution von Aromaten jedoch sehr oft über eine Addition an das $\pi$-Elektronensystem erfolgt, die geschwindigkeitsbestimmend ist, sind die Versuche mit Cyclohexan aussagekräftiger. Der $k_{\mathrm{H}} / k_{\mathrm{D}}$-Wert von 1,28 schließt eine Spaltung der CH-Bindung als geschwindigkeitsbestimmenden Schritt aus, deutet aber doch auf die Existenz eines sekundären Isotopeneffektes hin.

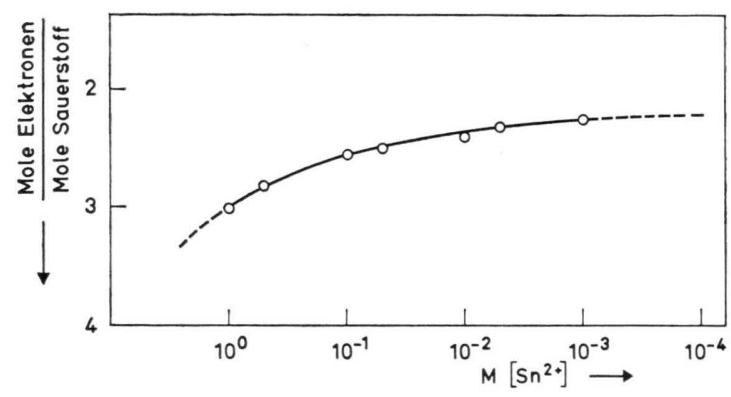

Abb. 3. Stöchiometrie von Elektronen: $\mathrm{O}_{2}$ im System $\mathrm{SnCl}_{2} /$ n-Hexanol. Versuchsbedingungen: s. "Methoden“.

Im Hinblick auf die Bedeutung der Oxen-Hydroxylierung als Modellmechanismus für die enzymatischen mischfunktionellen Oxygenierungen war es von großem Interesse, ob das Sauerstoffatom der eingeführten Hydroxylgruppe aus dem molekularen $\mathrm{O}_{2}$ stammt. Die Ergebnisse der Isotopenexperimente mit ${ }^{18} \mathrm{O}_{2}$ sind in Tab. 5 wiedergegeben:

\begin{tabular}{cccc}
\hline & \multicolumn{3}{c}{ Hydroxyacetanilide } \\
& $\begin{array}{ccc}o \\
{[\%]}\end{array}$ & {$[\%]$} & $p$ \\
& {$[\%]$} & 100 \\
\hline Einbau von ${ }^{18} \mathrm{O}$ & 74 & 98 & 100 \\
\hline
\end{tabular}

Tab. 5. Einbau von ${ }^{18} \mathrm{O}$ aus der Gasphase bei der Hydroxylierung von Acetanilid im System $\mathrm{Sn}^{2 \oplus} / \mathrm{HPO}_{4}{ }^{2} \odot / \mathrm{O}_{2}$. Versuchsbedingungen: s. "Methoden“.

Die Hydroxylgruppe in para-Stellung und auch in der gerade für den Oxen-Mechanismus charakteristischen meta-Stellung ist vollständig markiert.

Wesentlich schwieriger läßt sich die Stöchiometrie der Hydroxylierungen bestimmen. Die Kinetik der Reaktion und auch die relativ geringen Ausbeuten an Hydroxylierungs-Produkten bezogen auf die Menge des eingesetzten Zinn(II) -Komplexes lassen erkennen, daß der "aktive $\mathrm{O}_{2}$ “ zu einem überwiegenden Teil zu Wasser reduziert wird. Einer weiteren Erhöhung der Konzentration von Acetanilid sind durch dessen geringe Wasserlöslichkeit Grenzen gesetzt. 
Es wurde daher auf die Bestimmung des Produktes verzichtet und Alkohole in hoher Konzentration als Substrate angeboten. Bei diesen Versuchen ergab sich, daß Alkohole und Ketone gut als Komplexbildner für Zinn (II) -chlorid geeignet sind. Dadurch war es möglich, in fast wasserfreiem Medium zu hydroxylieren. Wie aus dem $o: m: p$-Verhältnis der $N$-Acetylaminophenole (Tab. 6) hervorgeht, ändert sich der Hydroxylierungs-Mechanismus dabei nicht.

\begin{tabular}{|c|c|c|c|c|c|}
\hline & \multirow[t]{2}{*}{ System } & \multirow{2}{*}{$\begin{array}{c}\text { Ausbeute } \\
{[\mu \mathrm{Mol}]}\end{array}$} & \multicolumn{3}{|c|}{ Hydroxyacetanilide } \\
\hline & & & $\begin{array}{c}0 \\
{[\%]}\end{array}$ & $\begin{array}{c}m \\
{[\%]}\end{array}$ & $\begin{array}{c}p \\
{[\%]}\end{array}$ \\
\hline \multirow{8}{*}{$\mathrm{SnCl}_{2}$} & Methanol & 0,76 & 54 & 32 & 14 \\
\hline & Äthanol & 0,96 & 52 & 36 & 12 \\
\hline & n-Propanol & 1,69 & 49 & 38 & 13 \\
\hline & n-Butanol & 1,28 & 48 & 37 & 15 \\
\hline & n-Hexanol & 0,87 & 48 & 38 & 14 \\
\hline & Dioxan & 0,15 & 38 & 33 & 29 \\
\hline & Aceton & 0,90 & 56 & 27 & 17 \\
\hline & Acetonitril & 2,62 & 58 & 28 & 14 \\
\hline
\end{tabular}

Tab. 6. Hydroxylierungen mit Zinn(II) -chlorid und $\mathrm{O}_{2}$ in organischen Lösungsmitteln.

$1 \mathrm{mMol} \mathrm{SnCl}{ }_{2} \cdot 2 \mathrm{H}_{2} \mathrm{O}$ und $20 \mathrm{mMol}$ Acetanilid in $20 \mathrm{ml}$ organischer Phase. Inkubationszeit $3 \mathrm{~h}$ unter Schütteln an der Luft. Nach dem Einengen wird der alkalisch gemachte Ansatz dreimal ausgeäthert. Die $N$-Acetyl-aminophenole extrahiert man aus Ammoniumacetat-gepufferter Lösung in Äther. Die angegebenen Werte sind Mittel aus zwei Versuchen.

Der Zinn(II) -Hexanol-Komplex erwies sich wegen der etwas geringeren Autoxydations-Geschwindigkeit als am besten geeignet für die Stöchiometrieversuche. Als Hydroxylierungs-Produkte findet man die entsprechenden Hexandiole. Trotz der sehr hohen Substratkonzentration tritt auch in diesem System neben der Hydroxylierung noch eine Reduktion des aktiven $\mathrm{O}_{2}$ zu Wasser ein. Die Stöchiometrie läßt sich jedoch ermitteln, wenn man das Verhältnis von eingesetztem Zinn(II)-Komplex zu $\mathrm{O}_{2}$-Verbrauch in Abhängigkeit von der Zinnkonzentration untersucht.

Bei Extrapolation auf unendlich kleine Zinn-Konzentration erhält man eine Stöchiometrie von 2 Molen Elektronen pro Mol Sauerstoff.

\section{Diskussion}

Die Ergebnisse dieser Arbeit bestätigen unsere früheren Vermutungen, daß die bei der Autoxydation von Zinn(II)-Komplexen zugesetzten Substrate nach einem neuen, einheitlichen Mechanismus hydroxyliert werden können. Obwohl bisher noch keine direkten Methoden zur Untersuchung des Me- chanismus dieser $\mathrm{O}_{2}$-Aktivierung gefunden wurden, lassen sich doch indirekt durch die Bestimmung der Hydroxylierungs-Produkte Hinweise auf den neuen Mechanismus erhalten.

Wasserstoffperoxid hemmt die Hydroxylierungen; es kann daher als Zwischenstufe bei der Entstehung des ,aktiven $\mathrm{O}_{2}$ “ ausgeschlossen werden. Die Eigenschaft des zweiwertigen Zinns bei der Oxydation zwei Elektronen abzugeben, sowie die Versuche zur Stöchiometrie zusammen mit den ${ }^{18} \mathrm{O}_{2^{-}}$Experimenten lassen nur die Deutung zu, daß der aktive $\mathrm{O}_{2}$ die Oxydationsstufe eines " $\mathrm{O}_{2}$-Atoms" besitzt. Dafür sprechen außerdem die schnelle Reaktion des aktiven $\mathrm{O}_{2}$ mit dem Zinn(II)-Komplex unter Wasserbildung und die hohe Reaktionsgeschwindigkeit gegenüber aliphatischen Verbindungen. Damit ist eine weitgehende Ähnlichkeit mit den Carbenoder Carbenoid-Reaktionen vorhanden, so daß der Begriff „Oxen". oder „Oxenoid"-Mechanismus für diese Reaktionen zutrifft.

Die Gesamtreaktion läßt sich demnach durch die folgende Gleichung beschreiben:

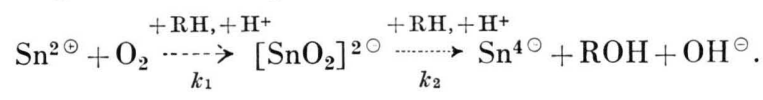

Aus dem Fehlen eines primären Isotopeneffektes läßt sich schließen, daß die Spaltung der CH-Bindung nicht im geschwindigkeitsbestimmenden Schritt verläuft. $k_{2}$ muß demnach größer sein als $k_{1}$. Diese Aussage wird gestützt durch die Beobachtung, daß Belichtung die Hydroxylierungs-Geschwindigkeit steigert. Die Strahlung muß daher den geschwindigkeitsbestimmenden Schritt beschleunigen, wahrscheinlich durch eine Anregung des $\mathrm{O}_{2}$-Moleküls. Mit Eisen(II) - und Kupfer (I) -Komplexen, bei den durch die besondere Elektronen-Konfiguration eine Spinumlagerung umgangen werden $\operatorname{kann}^{15}$, findet die Reaktion mit $\mathrm{O}_{2}$ wesentlich schneller statt, und man beobachtet keinen Einfluß von Licht auf die Hydroxylierung ${ }^{16}$.

Über die elektronische Struktur des „aktiven $\mathrm{O}_{2}{ }$ “ im System $\mathrm{Sn}^{2 \oplus} / \mathrm{HPO}_{4}{ }^{2 \ominus} / \mathrm{O}_{2}$ lassen sich noch keine Angaben machen.

Da der Oxen-Mechanismus in erster Linie als Modell für die mischfunktionellen Oxygenierungen untersucht wurde, bleibt noch seine Bedeutung für die enzymatischen Hydroxylierungen zu diskutieren. Als bisher einziger Mechanismus erfüllt die Hydroxy-

15 LL. L. Ingraham, Biochemical mechanisms, S. 68, Wiley, New York-London 1962.

16 V. UllRich, unveröffentlicht. 
lierung mit Zinn(II)-Komplexen und $\mathrm{O}_{2}$ die $\mathrm{Be}$ dingungen der $\mathrm{M}$ a s o $\mathrm{n}$ schen Gleichung.

Dennoch ist der aktive $\mathrm{O}_{2}$ mit Sicherheit nicht identisch mit der aktiven Species der Monooxygenasen, da die enzymatischen Hydroxylierungen elektrophil verlaufen ${ }^{8,17}$ und sich dadurch von den völlig unselektiven Substitutionen der Oxen-Hydroxylierung unterscheiden. Dieses abweichende Verhalten kann in einer unterschiedlichen Reaktivität des hydroxylierenden Teilchens bestehen. Eine solche $\mathrm{Zu}$ nahme der Selektivität könnte durch eine Komplexbildung und Stabilisierung des aktiven Sauerstoffs an dreiwertigem Eisen oder zweiwertigem Kupfer eintreten, die fast immer als essentielle Faktoren bei der enzymatischen $\mathrm{O}_{2}$-Aktivierung beteiligt

17 T. H. Elliott, R. C. C. TaO, and R. T. Williams, Biochem. J. 95, 70 [1965].

18 S. Fallab, Angew. Chem. 79, 500 [1967].

19 V. UlLRICH, in Vorbereitung. sind ${ }^{18}$. Die Existenz einer solchen elektrophilen „Oxenoid"-Hydroxylierung mit Eisen-Komplexen konnten wir kürzlich nachweisen ${ }^{19}$.

Die Bedeutung des Oxen-Mechanismus für die enzymatischen Reaktionen liegt vor allem in der Tatsache, daß eine Metallionen-katalysierte Zweielektronen-Reduktion des $\mathrm{O}_{2}$ nicht unmittelhar zu Wasserstoffperoxid führt, sondern über eine reak-

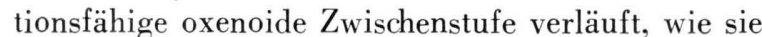
von GEORGE ${ }^{20}$ schon aus thermodynamischen Berechnungen postuliert worden ist.

Wir danken Herrn Dr. M. SenN von der Firma Bell \& Howell GmbH, Friedberg/Hessen, für die Durchführung der massenspektrometrischen Analysen.

$20 \mathrm{P}$. George, in: Oxidases and related redox system, T. King, H. S. Mason, and M. Morrison, Eds., Wiley, New York-London 1965.

Zur Koordinations-Isomerie (a-b-Isomerie) der Co-Alkyl-corrinoide

\title{
Partialsynthese und einige Eigenschaften des Co-Methyl-cobalamins a
}

\author{
Wilhelm Friedrich und Johann Peter Nordmeyer
}

\author{
Physiologisch-Chemisches Institut der Universität Hamburg \\ (Z. Naturforschg. 24 b, 588-596 [1969] ; eingegangen am 28. Oktober 1968)
}

\begin{abstract}
Chromatography and electrophoresis of the reaction products from $\mathrm{B}_{12 \mathrm{~s}}$ and methyl iodide yield the known Co-methyl-cobalamin and a new light sensitive corrinoid compound. The latter is very probably Co-methyl-cobalamin a with the methyl group "down". The absorption spectrum of this yellow corrinoid differs only slightly from that of Co-methyl-cobalamin in acid media and does not depend on $\mathrm{pH}$ at wavelengths higher than $300 \mathrm{~nm}$. The new corrinoid is thermolabile and on heating at $95{ }^{\circ} \mathrm{C}$ for several hours in $\mathrm{O}_{2}$-free aqueous medium changes nearly quantitatively to $\mathrm{Co}$ methyl-cobalamin. The last corrinoid, on the contrary, yields at $95{ }^{\circ} \mathrm{C}$ several per cents of Comethyl-cobalamin a. The reaction of $\mathrm{B}_{12}$ s with dimethyl sulfate yields beyond the mentioned products Co-methyl-cobalamin Nm (yellow; the absorption spectrum does not depend on $\mathrm{pH}$ and is identical with that of Co-methyl-cobalamin in acid media) and $\mathrm{B}_{12} \mathrm{Nm}$ (both corrinoids have a methyl group on N-3 of the 5,6-dimethylbenzimidazole moiety). The thermal isomerization of both Co-methyl-cobalamins is in accord with the observed formation of $\mathrm{Co}^{-14} \mathrm{CH}_{3}$-cobinamide on heating of $\mathrm{Co}^{-14} \mathrm{CH}_{3}$-cobalamin with $\mathrm{Co}-\mathrm{CH}_{3}$-cobinamide.
\end{abstract}

Aus diesem Laboratorium wurde über die a-b-Isomerie der Cyano-aquo-corrinoide berichtet ${ }^{1-5}$. Die beiden Isomeren erwiesen sich auf Grund ihrer Eigenschaften als Diastereoisomere mit der CNGruppe „unten“ in der a-Form bzw. „oben“ in der

1 W. FRIEDRICH, Biochem. Z. 342, 143 [1965].

2 W. Friedrich, Z. Naturforschg. 21 b, 138 [1966].

3 W. Friedrich, Z. Naturforschg. 21 b, 595 [1966].
b-Form, weshalb hier von einer Koordinations-Isomerie gesprochen werden kann. Kinetische Versuche ergaben, daß die beiden Formen unter sehr milden Bedingungen ineinander umgewandelt werden, wobei stets ein spezifisches Gleichgewicht $\mathbf{a}-$ Form $\rightleftharpoons \mathbf{b}$ -

\footnotetext{
4 W. Friedrich, H. Ohlms, W. Sandeck u. R. Bieganowski, Z. Naturforschg. 22 b, 839 [1967].

5 W. Friedrich u. M. Moskophidis, Z. Naturforschg. 23 b, 804 [1968].
} 